\title{
Le paysage linguistique du cimetière alsacien : un reflet diachronique et synchronique de la situation sociolinguistique.
}

\author{
Katharina Vajta* \\ Department of Languages and Literatures \\ University of Gothenburg, Sweden
}

\begin{abstract}
Résumé. Cette étude examine comment les choix linguistiques dans les cimetières alsaciens reflètent la situation sociolinguistique de la région et le changement de langue du germanique (allemand et alsacien) au français. Dans ce paysage linguistique (Landry \& Bourhis 1997), les inscriptions des épitaphes ont un rôle fonctionnel puisqu'elles comportent des informations personnelles sur les défunts (nom, dates, etc.), et aussi un rôle symbolique, de par le choix de langue qui pouvait être transgressif. En effet, en Alsace, région ayant basculé plusieurs fois entre France et Allemagne, le choix du français ou de l'allemand était censé suivre la langue du pouvoir. Mais ceci n'est pas toujours le cas. Ainsi, le choix du français pendant une période allemande pourra indiquer une prise de position identitaire en faveur de la France. Le choix de l'allemand, par contre, peut être considéré comme étant plutôt la conséquence de la tradition germanique de la région. De plus, noms et prénoms sont eux aussi marqués par les appartenances nationales. Aujourd'hui, le déclin des variétés germaniques est reflété par le choix général du français, l'allemand devenant exception.
\end{abstract}

\begin{abstract}
The linguistic landscape of the Alsatian cemetery : a reflection of the sociolinguistic situation in synchrony and diachrony. This study examines how language choices in Alsatian cemeteries reflect the sociolinguistic situation in the the region and the language shift from the Germanic varieties (German and Alsatian) to French. In this linguistic landscape (Landry \& Bourhis 1997), the inscriptions have a functional role, since they convey personal information about the departed (name, dates, etc.), and also a symbolic role, through transgressive linguistic choices. Indeed, Alsace has shifted several times between France and Germany, and linguistic choices were supposed to follow the language of the ruler. But this was not always the case, and the choice of French during a German period can indicate a positioning in favour of France. On the other side, the choice of German can be the consequence of the strong Germanic cultural tradition. Moreover, first names and family names were also concerned by the different national belongings. Today, the decline of German and Alsatian is reflected by the choice of French for the epitaphs and German has become an exception.
\end{abstract}

\footnotetext{
* Corresponding author: katharina.vajta@sprak.gu.se
} 


\section{Introduction}

L'Alsace, région située à la frontière franco-allemande, a basculé plusieurs fois entre la France et l'Allemagne. Devenue française à la fin de la guerre de Trente ans, au milieu du XVIIe siècle, elle reste cependant germanophone et le dialecte alsacien, dont la langue standard est l'allemand, est la langue vernaculaire. Au fur et à mesure, la République va cependant devenir plus stricte quant à la langue utilisée par ses citoyens alsaciens. Quand l'Alsace est cédée à l'Allemagne de par le traité de Francfort, à la fin de la guerre francoallemande de 1870-1871, l'allégeance à la France est pourtant claire ; l'usage du français devient aussi une façon de l'affirmer (Vogler 1993, 364). À la fin de la Première Guerre mondiale, l'Alsace redevient française et les Alsaciens sont alors supposés parler français à nouveau après presque cinquante ans sous régime linguistique et politique allemand. Pendant la Seconde Guerre mondiale, l'Alsace est annexée à l'Allemagne et elle redevient française à la Libération. (Pour un aperçu historique, voir par exemple Dollinger [1971] 1991, Vogler 1993.)

Ces aléas n'ont pas été sans laisser de traces, notamment dans les cimetières où les tombes et les épitaphes reflètent l'histoire de la région, à la fois sur les plans historique, linguistique et culturel. (Voir Vajta 2018.) Les langues présentes sont le français, le dialecte alsacien, langue quotidienne, et l'allemand, langue écrite de l'alsacien. Aujourd'hui, cependant, les variétés dialectales sont de moins en parlées et parallèllement l'allemand, leur langue standard, est moins présent. (Voir par exemple Tabouret-Keller 1988 ; Denis \& Veltman 1989 ; Vajta 2004 ; Huck 2013.) Il nous semble alors logique de pouvoir observer dans les cimetières de la région non pas tant l'alsacien, puisqu'il n'a pas de norme écrite, mais le français et l'allemand, et de pouvoir aussi y remarquer les traces de l'histoire régionale.

Ce travail se propose d'examiner comment les tombes et les monuments funéraires en Alsace représentent et reproduisent l'histoire linguistique et politique de la région. Comment les changements de langue s'y reflètent-ils? De quelles façons les langues y sont-elles utilisées ? Quelles sont les identités représentées ? Pour ce faire, nous allons tout d'abord présenter la notion de paysage linguistique (ou sémiotique) et discuter comment le cimetière peut être considéré comme étant un tel paysage. De plus, afin de compléter cette notion, nous référons ici également à celle d'hétérotopie de Foucault (2004). Nous présenterons ensuite la méthode de recherche et, plus précisément, les choix méthodologiques qui se sont imposés. Dans les parties suivantes, nous développerons la discussion portant sur les langues dans les épitaphes et les aspects identitaires que peuvent y dévoiler les choix linguistiques, que ce soit de par la langue elle-même, par les noms et prénoms, ou par une affirmation identitaire manifeste. Finalement, avant de conclure, un rapide état des lieux des épitaphes après 1945 témoignera du recul de l'allemand et de l'avancée du français.

\section{Le cimetière, un paysage linguistique}

Dans cette étude, le cimetière sera considéré comme un paysage linguistique, terme introduit par Landry et Bourhis en 1997, repris par de nombreux chercheurs, entre autres Gorter (2006, 2013), Shohamy \& Gorter (2009) et Coupland (2010), et depuis également dénommé paysage sémiotique (Jaworski \& Thurlow 2010), soulignant ainsi l'interaction entre le discours verbal écrit et d'autres modalités discursives (symboles, images, paysage urbain, etc.). (Pour une vue d'ensemble du champ, voir Shohamy 2019). Alors qu'elle date depuis une vingtaine d'années et semble être bien établie parmi les sociolinguistes anglosaxons, la notion de paysage linguistique semble pourtant avoir été moins utilisée dans le monde francophone. Un article en français de Kelleher (2017) en donne cependant un 
exposé général, relatant d'une part son histoire et son évolution depuis une orientation quantitative vers une approche descriptive et ethnographique, d'autre part présentant son utilité dans le cadre d'une méthodologie de recherche. Nous pouvons aussi noter, entre autres, les travaux de Bulot (2011) et de Lüdi (2010). En nous basant sur cette notion de paysage linguistique, notre ambition est donc aussi de contribuer à sa diffusion et de confirmer son intérêt. Le terme paysage sémiotique sera ici utilisé de manière pratiquement synonyme, tout en gardant en mémoire la dimension non-verbale qu'il apporte par rapport à paysage linguistique.

Selon Landry et Bourhis (1997, 25), le paysage linguistique d'un espace donné est principalement formé par les inscriptions, les affiches, les panneaux, les noms de rues et de magasins, et autres signes publics et visibles au sein de cet espace. Les langues - au pluriel car il s'agit en premier lieu d'un espace multilingue - y sont considérées comme ayant à la fois un rôle informationel et un rôle symbolique, et seront prises en compte dans leurs dimensions sociolinguistique et identitaire. En effet, les linguistic practices ou pratiques langagières se développent «dans des situations sociales qui impriment leurs marques à cette activité et à ses réalisations langagières » (Boutet 2016, 45). Gorter (2013, 191) observe que les études sur le paysage linguistique, dont le but est notamment de contribuer à de nouveaux savoirs sur le multilinguisme, peuvent concourir à de nouvelles connaissances dans le domaine des choix et des hiérarchies linguistiques, des langues en contact ou de politiques linguistiques. Les études effectuées ont porté sur des espaces linguistiques dans le monde entier, comme Taipei (Curtin 2014), la Frise et le Pays Basque (Cenoz \& Gorter 2006), Tokyo (Backhaus 2006, 2007), le Pays de Galle (Coupland 2010) ou la Bretagne et la Corse (Blackwood 2011). Cependant, comme le constate Pavlenko (2010, 133), les recherches sur les paysages linguistiques ont surtout focalisé sur les situations contemporaines en synchronie. Mais le rôle de la diachronie ne peut être négligé, ce que confirment Coupland \& Garrett $(2010,12)$ en constatant que la recherche dans ce domaine devraient davantage prendre en compte les contextes et les processus de développement historiques.

Dans chaque ville et village, que ce soit en Alsace ou ailleurs en Europe, nous trouvons un cimetière. Celui-ci constitue généralement un espace clairement délimité par un mur, une haie, ou un grillage, ouvert au public qui notera la démarcation entre extérieur et intérieur, marquée par une entrée en forme de grille ou de porte, une limite à franchir. (Voir aussi Rugg 2000.) Il s'agit d'un espace à part, séparé, délimité, et en même temps inclus dans le cadre d'une agglomération, soumis à une législation et à un réglement (municipal ou d'état), d'une sphère à la fois privée et publique : privée, car les visiteurs ont souvent une relation particulière et personnelle avec les défunts qui y reposent, publique, car le cimetière est d'habitude ouvert à qui veut y entrer, toute la journée, toute l'année.

Pour Foucault $(2004,16)$, le cimetière est un lieu autre, c'est-à-dire une hétérotopie : « le cimetière est certainement un lieu autre par rapport aux espaces culturels ordinaires, c'est un espace qui est pourtant en liaison avec l'ensemble de tous les emplacements de la cité, ou de la société, ou du village ». Cet espace devient «l'autre ville », un miroir à fonctionnement précis et particulier, reflétant la société mais d'une façon autre, altérée voire déformée. Le lieu dérange, dans bien des sens : il rappelle la disparition d'êtres chers et la fin de vie qui concerne tout être humain, et cela le rend paradoxalement banal - une banalité qui transforme ce lieu de mémoire en un endroit extra-ordinaire (Wright 2005, 69). Le cimetière est un lieu indispensable et inévitable; comme le remarque Foucault, " chaque individu, chaque famille se trouve avoir des parents au cimetière » $(2004,16)$. Il est d'autant plus exceptionnel qu'il est lié à une expérience finale individuelle et unique, et qu'il offre la possibilité de se souvenir et de commémorer, ce qui le transforme en espace sacré (Rugg 2000, 264 ; Francis 2003, 223). Rugg (2003) observe que les cimetières constituent un espace dynamique et changeant: ils reflètent l'histoire, la culture et la société environnante, et donnent l'occasion d'affirmer des identités, des différences, des 
relations personnelles : l'espace du cimetière réunit ainsi le politique, l'émotionnel et le culturel (Woodthorpe 2011, 260). Partant, les inscriptions sur les tombes fournissent au visiteur une mémoire culturelle ('cultural memory', Anderson et al. 2011). L'ensemble des inscriptions que nous y trouvons constitue le texte d'un paysage linguistique et sémiotique particulier, dont la qualité-même est caractéristique et délimite l'espace que constitue le cimetière et transforme celui-ci en un domaine qui n'est pas neutre, pouvant produire et reproduire identités, messages et significations. (Voir Blommaert 2013, 15.)

Le paysage linguistique sera ici compris dans l'acception de Gorter $(2013,190)$, c'est-àdire comme un texte où toute inscription visible, non seulement de langue écrite mais aussi de symboles, peut être étudié et interprété. Comme un territoire n'est jamais linguistiquement totalement homogène, l'usage des différentes langues se reflétera également dans ce paysage, où se manifesteront des indices révélant les relations entre les différents groupes linguistiques. Nous allons ici considérer le cimetière comme un espace semi-public où les épitaphes correspondent à une expression privée mais ouverte et visible à tous (Wright 2005, 61) afin de circonscrire comment les épitaphes reflètent le choix linguistique entre français et allemand, et de discuter les raisons possibles de ce choix ainsi que les identités révélées. Car dans un cimetière se trouvent quantité de messages et de signes, visibles à tout visiteur, que ce soit sur une plaque commémorative placée sur une tombe, l'épitaphe elle-même ou le panneau d'information à l'entrée. Ces textes doivent être étudiés dans leur contexte social, historique et culturel, et l'analyse doit aussi prendre en compte leur auteur - qui écrit quoi à qui, quand et pourquoi.

Or, Wright $(2005,60)$ observe que nous savons rarement avec certitude qui est l'auteur d'une épitaphe. En effet, l'auteur (ou l'agent) est souvent inconnu, même s'il s'agit le plus souvent de la famille ou de proches, peut-être même, parfois, de la personne décédée ellemême. L'auteur devient un acteur participant au paysage linguistique, où il tente de faire remarquer et observer son message. Ce qui nous amène à un deuxième participant: le public, qui dans un cimetière pourra consister de passants, de membres de la famille, de villageois, d'amis, de gardiens du cimetière, de fossoyeurs, de personnes jeunes ou âgées... Les inscriptions sur les tombes ne sont donc pas des messages délivrés dans un néant, mais des déclarations composant un discours qui sera lu, observé et compris par les passants (cf. Huebner 2009, 74). Ainsi, la perspective adoptée ici est celle du visiteur prenant part des inscriptions sans connaître les défunts mais pouvant situer les épitaphes dans le contexte historique et linguistique de l'Alsace.

\section{Choix méthodologiques}

Dans le cadre de cette étude, nous avons visité une quinzaine de cimetières en Alsace, dans des villages et des villes de taille moyenne situés surtout dans la partie nord de la région, plus germanophone que le sud. Tous étaient ouverts et accessibles au public, mais par égard envers les familles nous ne dévoilerons pas ici où se trouvent les différentes sépultures, à l'exception de quelques tombes bien connues du cimetière Sainte Hélène à Strasbourg. Comme c'est souvent le cas dans les études sur le paysage linguistique, l'appareil photo a été fort utile. Les pierres tombales ont été photographiées et les textes transcrits. En outre, à l'inscription sur la stèle vient parfois s'ajouter un texte complémentaire, par exemple sur une plaque commémorative.

Une approche quantitative s'avère d'emblée difficile, ce paysage linguistique étant sans cesse soumis à des conditions mettant sa durabilité à l'épreuve. Les pierres tombales sont souvent érodées et abîmées par le temps et les intempéries, cachées par la végétation, ou brisées, partiellement ou totalement. Selon l'état de conservation de la tombe, leur lecture est pénible ou difficile, voire impossible. Comme le remarque Coulmas $(2009,15)$, l'étude du paysage linguistique sous un angle historique doit fort souvent se contenter de vestiges, et l'état des tombes peut en effet parfois laisser à désirer. Inversement, elles ont parfois été 
restaurées ou réutilisées : il est parfois évident que l'inscription ou la pierre sont récentes, même si les dates sont d'une autre époque, ce qui amène alors la question de savoir quelle est la langue d'origine, par exemple si l'épitaphe a été traduite en français après avoir été auparavant en allemand, une question à laquelle il n'est guère possible ici d'apporter de réponse.

Pour ces raisons, cette étude se base sur des pierres tombales datant de toute évidence de l'époque de l'inscription. Les exemples donnés sont choisis parce qu'ils sont jugés représentatifs de caractéristiques observables en général dans tous les cimetières visités. L'analyse que nous proposons est qualitative et interprétative, et se base en premier lieu sur le texte verbal, en second lieu sur le contexte multimodal (Kress 2010; Scollon \& Scollon 2003).

\section{Langues et choix linguistiques dans les épitaphes}

En Alsace, les choix linguistiques possibles pour une épitaphe seront l'allemand, langue standard et écrite de l'alsacien, et le français. Les inscriptions en latin ne sont pas rares, mais il s'agira en général de phrases rituelles complétant le texte de l'épitaphe, par exemple «Requiescat in pace». Sur des tombes plus récentes, nous avons noté également la présence de l'anglais, de l'arabe, du portugais et de l'italien, notamment, reflétant une société plus mobile.

Les inscriptions suivent généralement un même modèle (Herat 2014 ; Bertrand 2005). Elles comportent alors le nom et prénom du défunt, ses dates de naissance et de décès, parfois des informations supplémentaires comme un lieu d'origine ou une citation biblique. Il n'est pas rare que les relations familiales soient précisées en quelques mots français (par exemple : épouse de, époux de...) ou allemands (Ehefrau, Ehemann...). Les dates pourront inclure le nom du mois de naissance ou de décès dans l'une ou l'autre langue, et de même une référence biblique sera parfois donnée, en français ou en allemand. Certaines tombes n'indiquent cependant qu'un nom de famille, ou un nom et prénom et les années de naissance et de décès, ne permettant alors pas d'identification linguistique. Il s'agit surtout de tombes plus récentes, les inscriptions semblant avoir tendance à devenir moins informatives qu'autrefois (Urbain 1988, 229 ; Vidor 2014, 355).

Le choix de l'une ou l'autre langue ne peut pas être considéré comme étant dû au hasard (Mytum 1994), même s'il ne se remarquera parfois que dans un discret geb. pour l'allemand geboren, ou née en français, pour indiquer un lieu de naissance ou un nom de jeune fille. Les inscriptions sont monophones ou monolingues en français ou en allemand, homophones avec un même message mais dans les deux langues, ou polyphones, à savoir différents messages dans les différentes langues. (Voir Coupland \& Garrett 2010, 11; Backhaus 2007, 90-103, Vajta 2018.) En principe, la langue des inscriptions doit correspondre à la langue du pouvoir, selon le principe du 'top-down'. Mais, remarque Lévy $(1929,372-373)$, cela n'est pas toujours le cas pendant la période allemande de 1871 à 1918, ce qui mettait parfois les autorités dans l'embarras, vu l'aspect sacré et inviolable d'un cimetière. Les transgressions linguistiques n'étaient pas rares et constituaient souvent une affirmation identitaire et idéologique. En outre, les langues ne sont pas toujours séparées et une même tombe comporte parfois des inscriptions et en français, et en allemand, et parfois l'inscription est en français alors qu'elle aurait dû être en allemand, ou inversement - selon le principe du 'bottom up'. Ceci dit, il faut tout de même considérer que dans les deux cas, chaque énoncé d'épitaphe se fait par rapport à l'idéologie linguistique promulguée par les autorités, que ce soit en l'acceptant ou en la refusant (Coupland 2010, 97). Cela nous amène à cinq catégories d'inscriptions différentes (Vajta 2018) :

celles qui correspondent à la langue officielle à la date du décès ; 
(B) celles qui sont monophones en français pendant une période allemande, à savoir de 1871 à 1918 ou de 1940 à 1945 ;

(C) celles qui sont monophones en allemand pendant une période française, à savoir avant 1871 , de 1918 à 1940 et après 1945 ;

(D) celles qui sont bilingues et polyphones ;

(E) celles ne présentant pas d'indication linguistique.

Les catégories $(A)$ et $(E)$ sont les moins conflictuelles. Il est possible de considérer la première $(\mathrm{A})$ comme un choix non marqué, peut-être dû au hasard, à de l'indifférence ou à une volonté de ne pas être en désaccord avec les autorités. Quant aux inscriptions de la catégorie (E), il se peut qu'elles soient une façon d'éviter un choix de langue et de prendre position ou, pour celles qui sont plus nouvelles, qu'elles fassent part de la tradition plus récente de ne pas inclure de renseignements à part le nom et éventuellement les dates appropriées.

Les catégories (B) et (C) comprennent les inscriptions marquant une transgression linguistique, mais sans doute pour des raisons différentes. Les inscriptions du groupe (C), monolingues en allemand pendant une période française, sont fréquentes avant 1871 et il est plausible que ce choix linguistique soit la conséquence de l'importante tradition germanique de la région, plutôt que d'une sorte de protestation. En effet, la politique linguistique des autorités françaises de l'époque n'était pas très rigoureuse et le français ne devient langue scolaire que dans la seconde moitié du XIXe siècle (Vogler 1993, 280-286 ; Lévy 1929, 196-320 ; Huck, Bothorel-Witz \& Geiger-Jaillet 2005, 9-10). Le fait d'utiliser une langue plutôt que l'autre n'avait alors pas de fonction symbolique. Par contre, la catégorie (B) semble plus problématique : le choix du français était alors clairement un signe d'opposition, qui pouvait entraîner certains risques.

Tableau 1

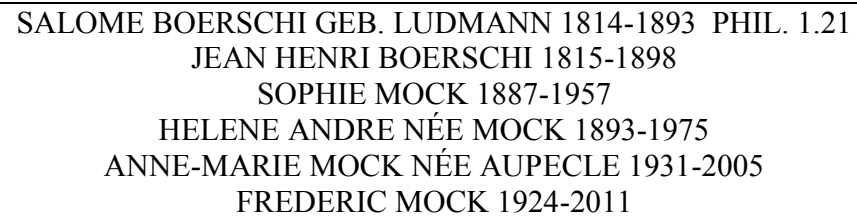

Le tableau 1 reproduit le texte d'une pierre tombale avec les inscriptions de six personnes différentes, un texte à caractère plutôt dépouillé, les principales informations étant les noms et les dates. Il n'en reste pas moins qu'il nous apporte certains renseignements intéressants. Les prénoms sont en français, ou dans une variante envisageable aussi dans les deux langues et s'intégrant phonologiquement dans l'une ou l'autre avec facilité (Salome, AnneMarie). Les accents aigu et grave de Hélène confirment qu'il s'agit bien de la forme française du prénom. Pour les prénoms masculins Jean Henri et Frédéric, les équivalents germaniques auraient été Hans ou Johannes, Heinrich et Friedrich. L'inscription de Salome, décédée pendant une période allemande, est en allemand, ce que signale l'abbréviation geb. placée avant le nom de jeune fille Ludmann, mais en dessous, celle d'Hélène, décédée en 1975, est en français, ce que signale née, de même celle d'Anne-Marie. Ces inscriptions sont donc comprises dans la catégorie (A) et, de plus, sur cette tombe, nous pouvons dans la transition de geb. à née observer le reflet du changement de langue à travers les générations.

Dans un même cimetière, le même nom de famille revient souvent d'une tombe à l'autre, et il semble vraisemblable qu'il puisse s'agir de générations différentes d'une même famille. Tel est le cas dans les inscriptions du tableau 2. L'inscription (a), où le 
prénom Cresentia serait plutôt germanique, est en allemand, pendant une période française. Les prénoms suivants dans (b) sont clairement français, de même née introduisant le nom de jeune fille de Béatrice, décédée pendant une période allemande. Le choix du français est confirmé trente ans plus tard dans (c), et ces deux inscriptions sont de la catégorie (B). Mais encore plus étonnante est l'inscription (d) avec l'usage du français pendant la Seconde Guerre mondiale. En effet, l'Alsace annexée était soumise à une germanisation totale et parler français signifiait prendre de grands risques. Ici, il est alors nécessaire de se poser la question de la date de l'inscription: peut-être a-t-elle été faite après la guerre ? Toujours est-il que dans la dernière inscription (e), la langue choisie n'est en fait révélée que par le mot Famille, qui l'inclut dans la catégorie (A). L'évolution observée à travers ces inscriptions de (a) à (e) témoigne ainsi du changement de langue de l'allemand au français, et accessoirement, de la tendance à écourter le texte des épitaphes.

Tableau 2

\begin{tabular}{|l|l|l|l|l|}
\hline \multicolumn{1}{|c|}{ (a) } & \multicolumn{1}{|c|}{ (b) } & \multicolumn{1}{c|}{ (c) } & \multicolumn{1}{c|}{ (d) } & \multicolumn{1}{c|}{ (e) } \\
\hline Hier ruht in Gott & Jean Thibaut & Celestin Wach & Ici repose en & Famille Wach \\
Cresentia Wach & Wach & $1828-1911$ & Dieu & Raymond 1897- \\
geb. den $24^{\mathrm{T}}$ & $1799-1847$ & Elisabeth Wach & Alexandre Wach & 1960 \\
April & Béatrice Wach & née Kormann & $1870-1943$ & Julia1902-1994 \\
1818 & née Metz & $1829-1911$ & & Josiane1961- \\
gest. den 6 ${ }^{\mathrm{T}}$ Iuni & $1805-1881$ & R.I.P. & & 2003 \\
1855 & & & Charlotte \\
Alt 37 Jahr & & & & $1967-2013$ \\
\hline
\end{tabular}

La première indication d'un changement de langue de l'allemand au français semble donc être les petits mots signalant les dates et les noms de jeune fille. Cela n'empêche qu'un texte complémentaire en allemand reste fréquent, par exemple une phrase rituelle comme Ruhe sanft ou un verset biblique. Une utilisation parallèle des deux langues peut ainsi être observée jusqu'à la fin du XXe siècle, période après laquelle les occurrences de l'allemand sont rares, indiquant que ce choix tombe en désuétude.

\section{Noms et prénoms}

Choisir comment appeler une part du monde a une portée sur la réalité et comme l'observe Boutet : «nommer le monde, ici des personnes, n'est pas un acte anodin » $(20016,19)$. En Alsace, l'évolution des noms et surtout des prénoms révèle la transition d'une culture germanique à une culture française et nommer le monde devient aussi un acte politique et idéologique. Urbatsch $(2014,463)$ constate que "naming children is a very powerful signal" et Ainiala \& Östman $(2017,4)$ observent que "social values [...] have great importance in the selection of a first name: national background, mother tongue, religious convictions [...] affect name giving". Car contrairement aux noms de famille qui sont traditionnellement des anthroponymes héréditaires, les prénoms font l'objet d'un choix. Ce choix est en général celui des parents ou de la famille proche, mais il peut faire l'objet de certaines conditions ou restrictions. Ainsi, pendant la période allemande de 1871 à 1918 ainsi que pendant la Seconde Guerre mondiale, les prénoms français sont interdits (Lévy 1929, 365, 434; Philipps [1975] 1986, 133-134).

Les noms de famille sont généralement germaniques et ce sont alors en premier lieu les prénoms qui reflètent l'histoire bilingue de la région. Si la jeune génération se prénomme Albert, Ernestine et Louise, Charles ou Louis, celle des parents s'appellera Albrecht, Wilhelmine, Karl, Ludwig. Le changement de prénom selon la langue en vigueur se remarque spécialement dans l'inscription de Françoise Hauser, dont le prénom est français, 
et par sa forme et par sa signification (voir tableau 3). Son décès a lieu en 1861, alors que l'Alsace est française, et son épitaphe l'identifie comme étant "Epouse de Louis Hentz ». Par contre, ce même Louis sera appelé Ludwig et son épitaphe sera en langue allemande lorsqu'il décède en 1891. Ces prénoms suivent loyalement la langue du pouvoir, même si une occurrence de ce qui est probablement dû à une insécurité linguistique peut être observée dans le nom du mois octobre qui en allemand aurait dû être Oktober : le mois est en français malgré le contexte en allemand, mais la lettre initiale est majuscule, comme le veut la graphie allemande.

\section{Tableau 3}

\begin{tabular}{|c|}
\hline Ici repose \\
Françoise Hausser \\
Epouse de \\
Louis Hentz \\
née le 23 mars 1811 \\
décédée le 8 novembre \\
1861 \\
Ludwig Hentz \\
Geboren \\
den 10. Octobre $($ sic $) 1810$ \\
Gestorben \\
den 22 juli 1891 \\
\hline
\end{tabular}

Pendant la première moitié du XIXe siècle, le choix d'un prénom allemand était courant, la politique linguistique des autorités françaises n'étant pas très stricte. Nous trouverons donc, avant 1871, des prénoms dans les deux langues. L'évolution vers le français semble se faire de façon plutôt volontaire et le choix du français semble devenir délibéré, comme dans les épitaphes du tableau 4.

Tableau 4

\begin{tabular}{|c|c|}
\hline $\begin{array}{c}\text { Familien } \\
\text { Hier ruht in Gott } \\
\text { Theobald Schmitt } \\
\text { geb. 14. Juli } 1845 . \\
\text { gest. 13. April } 1913 . \\
\text { Ruhet sanft. }\end{array}$ & $\begin{array}{c}\text { Grabstätte } \\
\text { Hier ruht in Gott } \\
\text { Anna Schmitt } \\
\text { geb. Johannes } \\
\text { geb. 9. März } 1847 . \\
\text { gest. } 29 \text { Jan. } 1903 .\end{array}$ \\
\hline \multicolumn{2}{|c|}{$\begin{array}{c}\text { Thiebaut Schmitt } \\
1904-1937\end{array}$} \\
\hline
\end{tabular}

Dans cette famille Schmitt, les épitaphes de ceux qui sont probablement les grands-parents de Thiebaut sont non seulement en allemand, mais aussi en écriture gothique (ici en italique) soulignant ainsi la tradition germanique (Spitzmüller 2012, 261). Les prénoms Theobald et Anna sont en allemand, mais Thiebaut, né pendant une période allemande, est clairement français et transgressif. Nous pouvons ici envisager plusieurs possibilités. Peutêtre le prénom était-il en allemand dans les registres d'état-civil, mais que l'on utilisait Thiebaut. Peut-être le prénom a-t-il été changé lorsque l'Alsace est redevenue française en 1918 et qu'on l'a alors inscrit en français sur la tombe. Finalement, il se peut aussi que l'on ait réussi à nommer son enfant Thiebaut même si cela était contre les règles et que les autorités aient fermé les yeux. Toujours est-il que cette famille se révèle avoir fait le choix d'abandonner l'allemand, ce qui se reflète dans les épitaphes et les prénoms, et même dans la police d'écriture de l'inscription. 


\section{Affirmations identitaires}

Les inscriptions ne suivent donc pas toujours la langue du pouvoir, malgré la juridiction. Tout semble indiquer deux explications plausibles : la tradition et le désir d'affirmer une identité. En effet, l'effet d'une tradition culturelle et linguistique germanique peut ne laisser qu'une option naturelle quant à la langue d'une épitaphe, amenant ainsi le choix de l'allemand pendant une période française ; ce sont les inscriptions de la catégorie (C). Ce choix devient alors en réalité un choix non marqué bien que transgressif, puisqu'il apparaît comme davantage causé par une tradition que par le souhait d'affirmer une identité germanique par rapport à une identité française. Par contre, le choix du français pendant une période allemande (catégorie B) est transgressif et marqué, car il était en fait interdit (Lévy 1929, 365, 434 ; Philipps [1975] 1986, 133-134).

Ainsi, le choix du français pour Barbe Meyer (voir tableau 5 a), Jean Ignace Hatterer (voir tableau 5 b) et Laurent Schmitt (voir tableau 5 c) est manifeste. Lévy (1929, 372-373) note que cette pratique linguistique n'était pas inaccoutumée à l'époque, et décrit comment les autorités germaniques, tout en la répprouvant, ne réussissaient pas vraiment à faire suivre leur politique linguistique, surtout dans les cimetières. Il était cependant difficile pour les autorités d'intervenir contre les familles, même si les valeurs signalées poussaient les limites de l'acceptable : l'agent auteur de l'épitaphe n'est pas toujours identifiable, la mort commande le respect et le cimetière est généralement considéré comme un espace sacré (Wright 2005, 60).

Tableau 5

\begin{tabular}{|c|c|c|}
\hline (a) & (b) & (c) \\
\hline Ici reposent en Dieu & Ici repose en Jésus-Christ & Laurent Schmitt \\
Barbe Meyer & Jean Ignace Hatterer & époux de Véronique \\
née Fohrer & décédé & Rauscher \\
$1800-1880$ & le 10 décembre 1878 & décédé le $21 \mathrm{X}^{\mathrm{BRE}}$ \\
Antoiner Meyer & âgé de 56 ans & 1872 \\
$1829-1884$ & R.I.P. & À l'âge de 57 ans. \\
Barbe Fohrer & & Req. in pace! \\
$1824-1892$ & & \\
\hline
\end{tabular}

Dans certaines inscriptions, le français est utilisé dans des phrases rituelles («Ici repose ») ou indiquant une relation familiale (" époux de »). Ceci était sans doute estimé moins subversif et controversé que les inscriptions comprenant une allusion au régime français, par exemple un grade militaire ou un métier, une décoration civile ou militaire ou une fonction d'élu comme maire ou député. De ce fait, les inscriptions de la figure 6 sont toutes transgressives et leur contenu signale une prise de position idéologique et une identité française : capitaines, sous-lieutenant, chevalier et officiers de la Légion d'honneur, docteur, directeur, député et maire... Expliciter ces titres, qui indiquent un service accompli dans la société et l'armée françaises, parfois au prix de sa vie, signale clairement une prise de position pour la France.

En effet, l'ensemble des inscriptions sur le monument funéraire de la famille Epp (tableau 6 a-b) atteste une tradition de père en fils défendant les couleurs françaises, soulignée par la cocarde tricolore indiquant que la tombe est prise en charge par l'association du Souvenir français. Les inscriptions indiquent non seulement une fonction dans l'armée et les dates de naissance et de décès, mais aussi les décorations attribuées (Légion d'honneur), et rapportent pour deux des défunts les circonstances de leur disparition, à savoir en combattant pour la France. 
Quant à l'épitaphe sur la tombe du maire et député E. [Emile] Küss (tableau 6 c), elle relate en en quelques mots et en français une histoire à la fois personnelle, régionale et nationale. Elle indique que Küss est décédé à Bordeaux, et implique qu'il a donc fait le choix de quitter l'Alsace devenue allemande en 1871. De plus, le décès dramatique de Küss rajoute encore un aspect patriotique - il est mort d'une crise cardiaque en apprenant que la France cédait l'Alsace à l'Allemagne à la fin de la guerre. L'agent auteur de l'épitaphe est ici explicite : "Ses compatriotes », en d'autres termes, ceux qui partagent ses convictions. L'emplacement même de la tombe, bien en vue à un croisement d'allées, ainsi que sa taille imposante, la transforment quasiment en une déclaration publique d'une identité française, en même temps que le grès rose de la stèle la rattache à l'Alsace où cette pierre des Vosges est fréquemment utilisée et a notamment servi à la cathédrale de Strasbourg. Comme le constate Huebner \& Phoocharoensil (2017, 107), un tel ensemble d'éléments confère une signification à un monument et l'inclut dans un processus sémiotique où le texte verbal, la matérialité et la localisation dans l'espace contribuent à la construction de sens et confirme l'importance non seulement de la personne mais aussi de son rôle et de ses convictions.

Tableau 6

\begin{tabular}{|l|l|l|l|}
\hline \multicolumn{1}{|c|}{$(\mathbf{a})$} & \multicolumn{1}{|c|}{ (b) } & \multicolumn{1}{c|}{ (d) } \\
\hline C.A. EPP & Alfred Epp & E. Küss & Charles Feibel \\
Capitaine & Capitaine de cavalerie en & Professeur à la & Directeur de banque \\
16 d'artillerie & retraite & faculté de & 1848-1888 \\
pontonniers & Chevalier de la légion & médecine & Aimé Bohn \\
Chevalier & d'honneur & MAIRE DE & Docteur en médecine \\
de la légion & 4 déc 1860 - 1 déc 1912 & STRASBOURG & 1850-1894 \\
d'honneur & Sous lieutenant 204 & Député à & R.I.P. \\
tombé sur les & Alfred Epp & l'Assemblée & \\
remparts & 24 sept 1892 - 25 sept & nationale & \\
de Strasbourg & 1915 & Strasbourg 1815 - & \\
le 8 septembre 1870 & Chevalier de la légion & Bordeaux 1871 & \\
à l'âge de 41 ans & d'honneur & & \\
& Croix de guerre & Ses compatriotes & \\
& Tué à l'ennemi le 25 sept & & \\
& 1915 au nord d'Arras & & \\
& \multicolumn{2}{|l}{} \\
\end{tabular}

\section{Présence de l'allemand après 1945}

L'Alsace, redevenue française à sa libération en 1945, fait l'objet d'une politique linguistique sévère de la part des autorités françaises en vue de favoriser l'usage du français qui doit remplacer l'allemand. L'influence germanique subsiste cependant, surtout dans le nord, où il semble donc plus probable de trouver des inscriptions funéraires en allemand. Effectivement, déjà à l'entrée du cimetière de Lauterbourg, un panneau bilingue français allemand sur lequel est stipulé le règlement du cimetière selon le " décret du 23 Prairial an XII », en allemand "Gemäss Dekret vom 23 ten Prairial Jahr XII", confirme que l'allemand est encore présent dans cette région.

Nous trouvons de nombreuses inscriptions en allemand datant des années 1950, mais les occurrences diminuent pour plus ou moins cesser à la fin du XXe siècle. Mais même si les indications des dates de naissance et de décès ainsi que des noms de jeune fille sont alors introduites par le français né ou née et décédé ou décédée, ces inscriptions sont souvent complétées par un verset biblique ou un autre texte, poème, mots d'affection de la famille ou autre. Celui-ci sera alors souvent en allemand, comme dans les exemples du tableau 7. 
Dans les exemples (b), (c) et (d) de ce tableau 7, les textes allemands sont inscrits sur une plaque commémorative posée sur la tombe. L'exemple (a) comporte un texte en allemand gravé sur la stèle même, et atteste un bilinguisme français - allemand, d'une part par «née » avant le nom de jeune fille, d'autre part par l'expression en allemand. Il est à noter également que le prénom Theophil a une forme germanique, vu l'absence de $<\mathrm{e}>$ final, et l'absence d'accents sur les deux prénoms vient corroborrer cette présomption. Ensuite, l'exemple (b) présente un choix du français pour indiquer les dates et les noms de jeune fille, alors que le verset biblique est cité en allemand. L'exemple (c), par contre, ne donne pas d'indication linguistique de par les dates et les noms, ce qui implique une absence (volontaire ou non) de choix linguistique, mais il comporte aussi un texte commémoratif en allemand. Le dernier exemple (d) présente un bilinguisme français - allemand, où le français est utilisé dans la rubrique «Famille » et sur l'une des plaques commémoratives, adressée « À mon cher frère », alors que celle adressée par les enfants à leur père est en allemand, ce qui illustre clairement la présence synchronique des deux langues. La datation du texte allemand n'est cependant pas certaine, puisqu'il peut remonter soit à 1976 , soit à 2008 , selon à qui il se rapporte.

Tableau 7

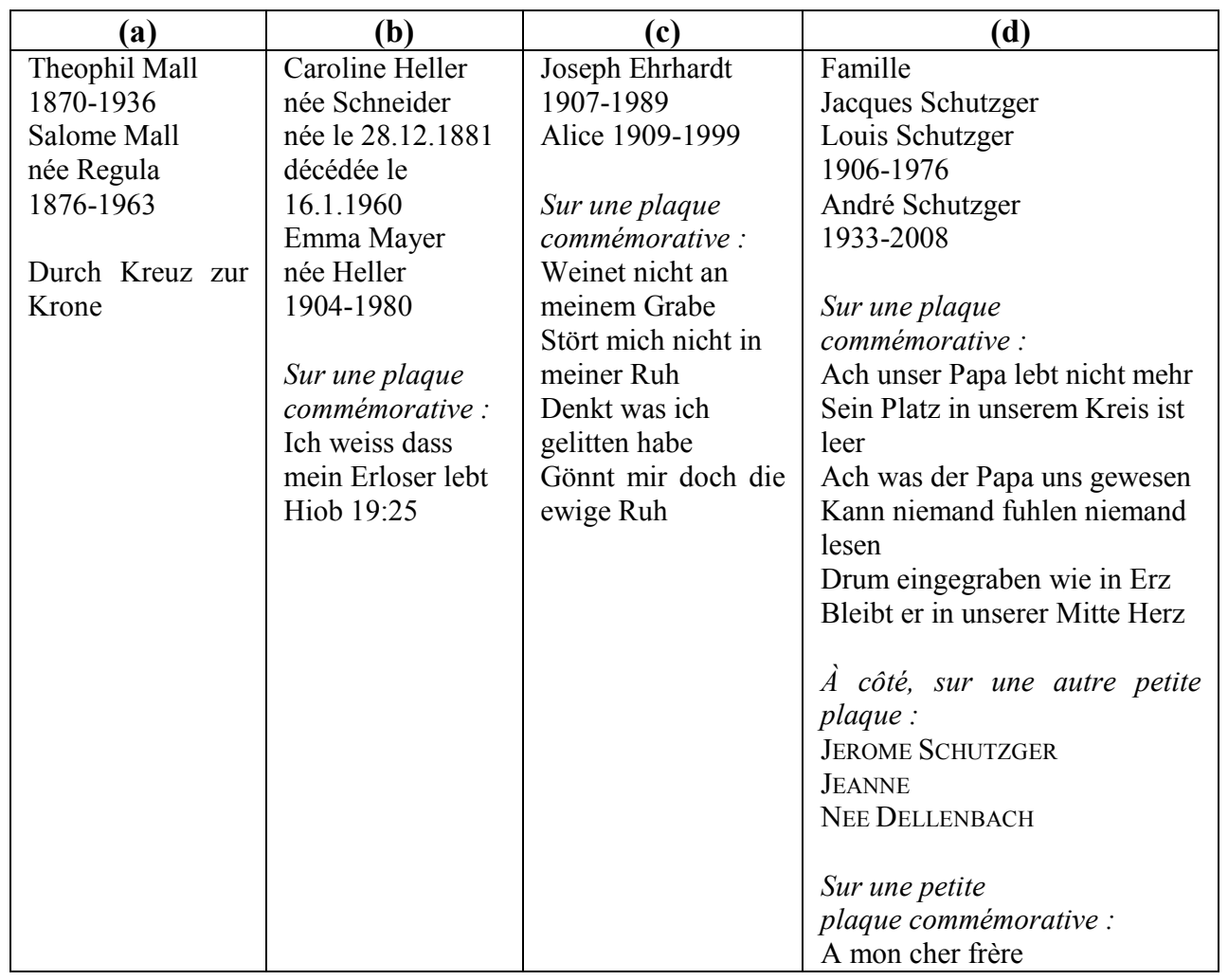

Dans leur ensemble, les exemples du tableau 7 illustrent comment le français vient à successivement suppléer l'allemand dans les inscriptions funéraires. Ce dernier n'est ici utilisé que dans des textes complémentaires (plaques commémoratives, citations bibliques) et non pas pour indiquer une date ou un nom, mais il est malgré tout présent dans un texte à caractère privé ou religieux. Or, le fait que geb. ou geboren, et gest. ou gestorben en allemand soit remplacés par né(e) et décédé(e) se révèle être un premier signe de 
changement langue dans les épitaphes en Alsace, tandis que les phrases rituelles ou les références à la Bible semblent se maintenir en allemand ultérieurement. En effet, les textes complémentaires sont habituellement en français lorsque l'inscription est bilingue ou en français, parfois en allemand, mais une inscription monolingue en allemand n'aura guère de texte complémentaire en français.

Cette évolution dans le choix de langue dans les épitaphes est illustrée très concrètement dans le cimetière du petit village de Hunspach. Ici, contrairement aux autres cimetières où les tombes de différentes dates et époques sont plus ou moins mêlées, les tombes se répartissent sur quatre carrés différents (voir tableau 8). Le premier est celui des tombes les plus anciennes, le plus souvent antérieures à 1930 et toutes en allemand; la plupart des inscriptions y sont en écriture gothique. Le cimetière a ultérieurement été agrandi par un deuxième espace carré qui comporte des inscriptions dans les deux langues, souvent monolingues en allemand encore après 1945, le français étant introduit progressivement dans des inscriptions bilingues ou, plus nouvellement, monolingues en français. Le troisième carré est celui des tombes les plus récentes et les inscriptions en français y sont très clairement celles dont le nombre domine, ce qui sera sans doute le cas également dans le dernier carré, encore non utilisé. En fait, selon le modèle de Haugen (1953, 370-371), ces différentes parties correspondent aux différents stades du changement de langue: la répartition des tombes, qui s'est faite naturellement selon la nécessité d'agrandir le cimetière, en reflète les différentes étapes et propose ainsi un parallèle au changement de langue en Alsace dont il devient quasiment une métonymie.

Tableau 8

\begin{tabular}{|l|l|}
\hline $\begin{array}{l}\text { Carré II : } \\
\text { Bilingue français - } \\
\text { allemand, polyphone }\end{array}$ & Carré IV : non utilisé \\
\hline $\begin{array}{l}\text { Carré I : Allemand } \\
\text { monolingue et } \\
\text { monophone }\end{array}$ & $\begin{array}{l}\text { Carré III : Français } \\
\text { monolingue et } \\
\text { monophone }\end{array}$ \\
\hline
\end{tabular}

\section{Conclusion}

Le changement de langue menant à ce qui est aujourd'hui en Alsace un usage quasigénéralisé du français se remarque surtout à partir du milieu du XIXe siècle. C'est en effet à cette époque que les mots né(e) ou décédé(e) introduisant les dates de début et de fin de vie, ou un nom de jeune fille, sont inclus dans les inscriptions et remplacent l'allemand geb. ou geboren, et gest. ou gestorben. Une même stèle, où né succède à geb., peut afficher le changement de langue du français à l'allemand d'une génération à l'autre, rendant la tombe bilingue. Les textes complémentaires (phrases rituelles, versets bibliques, poésies) sont souvent encore en allemand. Tout en déclinant, celui-ci est cependant présent jusqu'à la fin du XXe siècle.

Après 1871, le français devient une manière d'affirmer une identité non-allemande et de défier la nouvelle appartenance nationale imposée à la fin de la guerre par le Traité de Francfort : le cimetière devient un lieu de résistance, où le français constitue un choix marqué et délibéré, contrairement à la tradition germanique pourtant très importante, mais qui correspond davantage à un choix s'alignant dans une tradition linguistique qu'à une prise de position. Dans les différents cimetières, l'allemand et le français figurent plus ou 
moins en parallèle dans les épitaphes jusque vers le milieu du XXe, quand le français commence à devenir la langue dominante.

Les choix linguistiques que présentent les tombes reflètent à la fois l'histoire de la région et la situation sociolinguistique actuelle. Ces choix, conscients ou non, se révèlent notamment dans les noms des défunts, les épitaphes et les textes complémentaires souvent apposés aux tombes. Mais la visibilité des langues ne réfère pas simplement à une réalité dans la vie des vivants, elle contribue à la construction symbolique d'un nouvel espace, comme le remarque Tufi $(2016,101)$. Ces voix qui s'approprient une part de l'espace expriment un message à la fois individuel, collectif - mais non pas unanime - et de plus, public et visible aux visiteurs. Elles composent de la sorte un ensemble polyphonique où sont transmis des messages identitaires, idéologiques et politiques. C'est ainsi que le cimetière devient un lieu autre, une hétérotopie, où ces voix se superposent en diachronie et en synchronie et où s'expriment, plus ou moins explicitement, différentes identités.

Dans ce paysage sémiotique, les choix linguistiques constituent l'écho des différentes appartenances nationales et de cette façon les épitaphes contribuent au maintien d'une mémoire collective. Les épitaphes n'ont pas seulement un rôle fonctionnel, permettant de trouver une certaine tombe. Elles remplissent aussi une fonction symbolique, d'une part historiquement, comme un signe d'opposition à un pouvoir allemand ou en transmettant des idées et des convictions, d'autre part, aujourd'hui, comme un miroir des tournants historiques de la région.

\section{Références bibliographiques}

Ainiala, Terhi \& Jan-Ola Östman (2017). Introduction. Socio-onomastics and pragmatics. Socio-onomastics. The pragmatics of names, 1-18. Ed. Terhi Ainiala and Jan-Ola Östman. Amsterdam: John Benjamins Publishing Company.

Anderson, Keith A., Christine L. Sielski, Elizabeth A. Miles \& Alexis V. Dunfee (2011). Gardens of stone: Searching for evidence of secularization and acceptance of death in grave inscriptions from 1900-2009. Omega 63 (4), 359-371.

Backhaus, Peter (2006) Multilingualism in Tokyo: A Look into the Linguistic Landscape, International Journal of Multilingualism 3 (1), 52-66.

Backhaus, Peter (2007). Linguistic Landscapes. A Comparative Study of Urban Multilingualism in Tokyo. Clevedon: Multilingual Matters Ltd.

Bertrand, Régis (2005). " "Que de vertus... » Les épitaphes édifiantes des débuts du XIXe siècle." Les narrations de la mort, 241-255. Eds. Bertrand Régis, Anne Carol \& Jean-Noël Pelen. Aix-en-Provence: Publications de l'Université de Provence.

Blackwood, Robert (2011). The linguistic landscape of Brittany and Corsica: A comparative study of the presence of France's regional languages in the public space. Journal of French Language Studies 21 (02), 11-130.

Blommaert, Jan (2103). Ethnography, Superdiversity and Linguistic Landscapes. Chronicles of Complexity. Bristol, Buffalo, Toronto: Multilingual Matters.

Boutet, Josiane (2016). Le pouvoir des mots. (Nouvelle édition.) Paris, La Dispute/Snédit.

Bulot, Thierry (2011). Sociolinguistique urbaine, Linguistic Landscape Studies et scripturalité : entre convergence(s) et divergence(s). Cahiers de Linguistique, EME Edition, 37 (1), 5-15.

Cenoz, Jasone \& Duck Gorter (2006). Linguistic Landscape and Minority Languages. International Journal of Multilingualism 3 (1), 67-80. 
Coulmas, Florian (2009). Linguistic landscaping and the seed of the public sphere. Linguistic Landscape. Expanding the Scenery, 13-24. Eds. Elana Shohamy \& Durk Gorter. New York: Routledge.

Coupland, Nikolas (2010). Welsh Linguistic Landscapes 'From Above' and 'From Below'. Semiotic Landscapes. Language, Image, Space, 77-101. Eds. Adam Jaworski and Crispin Thurlow. London: Continuum.

Coupland, Nikolas \& Peter Garrett (2010). Linguistic landscapes, discursive frames and metacultural performance: the case of Welsh Patagonia. International Journal of the Sociology of Language 205, 7-36.

Curtin, Melissa (2014). Mapping cosmopolitanisms in Taipei: toward a theorisation of cosmopolitanism in linguistic landscape research. International Journal of the Sociology of Language 228, 153-177.

Denis, Marie-Noëlle \& Calvin Veltman (1989). Le déclin du dialecte alsacien. Strasbourg : Association des publications près les Universités de Strasbourg.

Dollinger, Philippe, ed. ([1971]1991). Histoire de l'Alsace. Toulouse: Privat.

Foucault Michel (2004). Des espaces autres. Empan 2004/2, 54, 12-19.

Francis, Doris (2003). Cemeteries as cultural landscapes. Mortality 8 (2), 222-227.

Gorter, Durk (2006). Introduction: The Study of the Linguistic Landscape as a New Approach to Multilingualism. International Journal of Multilingualism 3(1), 1-6.

Gorter, Durk (2013). Linguistic Landscapes in a Multilingual World. Annual Review of Applied Linguistics 33, 190-212.

Haugen, Einar (1953). The Norwegian Language in America. Philadelphia, Pennsylvania: University of Pennsylvania Press.

Herat, Manel (2014). The final goodbye: The linguistic features of gravestone epitaphs from the nineteenth century to the present. International Journal of Language Studies 8 (4), 127-150.

Huebner, Thom (2009). "A framework for the linguistic analysis of linguistic landscapes". Linguistic Landscape. Expanding the Scenery, 70-87. Eds. Elana Shohamy \& Durk Gorter. New York: Routledge.

Huebner, Thom \& Supakorn Phoocharoensil (2017). Monument as semiotic landscape. The contested historiography of a national tragedy. Linguistic Landscape 3 (2), 101-121.

Huck, Dominique (2013). Dialectes et allemand en Alsace. Histoire sociale des langues de France. Ed. Georg Kremnitz, 397-410. Rennes, Presses universitaires de Rennes.

Huck, Dominique, Arlette Bothorel-Witz \& Anemone Geiger-Jaillet (2005). Report on the linguistic situation in Alsace (France). www.eurac.edu/languagebridges (accès internet le 5 août 2015).

Jaworski, Adam \& Crispin Thurlow (2010). Introducing Semiotic Landscapes. Semiotic Landscapes. Language, Image, Space, 1-40. Eds. Adam Jaworski \& Crispin Thurlow. London, New York: Continuum.

Kelleher, William (2017). Les Linguistic Landscape Studies. Langage et Société 160-161, $337-347$. 
Kress, Gunther (2010). Multimodality. A social semiotic approach to contemporary communication. London, New York: Routledge.

Landry, Rodrigue \& Richard Y. Bourhis (1997). Linguistic landscape and ethnolinguistic vitality. An empirical study. Journal of Language and Social Psychology 16 (1), 2349.

Lévy, Paul (1929). Histoire linguistique d'Alsace et de Lorraine. Tome II: De la Révolution française à 1918. Paris : Société d'édition Les Belles Lettres.

Lüdi, Georges (2010). L'analyse du paysage linguistique comme instrument pour analyser la gestion des langues dans les entreprises. Le plurilinguisme au travail entre la philosophie de l'entreprise, les représentations des acteurs et les pratiques quotidiennes, 87-106. Ed. Georges Lüdi. Bâle : Institut für Französische Sprachund Literaturwissenschaft der Universität Basel.

Mytum, Harold (1994). Language as Symbol in Churchyard Monuments: The Use of Welsh in Nineteenth- and Twentieth-Century Pembrokeshire. World Archaeology 26 (2), 252-267.

Pavlenko, Anet (2010). Linguistic Landscape of Kyiv, Ukraine: A Diachronic Study. Linguistic Landscape in the City, 133-150. Eds. Elena Shohamy, Eliezer BenRafael \& Monica Barni. Bristol: Multilingual Matters.

Philipps, Eugène ([1975] 1986). Les luttes linguistiques en Alsace jusqu'en 1945. Strasbourg: Société d'édition de la Basse-Alsace.

Rugg, Julie (2000). Defining the place of burial: What makes a cemetery a cemetery? Mortality 5 (3), 259-275.

Rugg, Julie (2003). Introduction: Cemeteries. Mortality 8 (2), 107-112.

Scollon, Ron, and Suzie Wong Scollon (2003). Discourses in Place. Language in the Material World. London: Routledge.

Shohamy, Elana (2019). Linguistic Landscape after a Decade: An Overview of Themes, Debates and Future Directions. Expanding the Linguistic Landscape. Linguistic Diversity, Multimodality and the Use of Space as a Semiotic Resource, 25-37. Ed. Martin Pütz. \& Neele Mundt. Bristol: Multilingual Matters.

Shohamy, Elana \& Duck Gorter, eds (2009). Linguistic Landscape. Expanding the Scenery. New York, London: Routledge.

Spitzmüller, Jörgen (2012). Floating ideologies: Metamorphoses of graphic "Germanness". Orthography as social action. Scripts, spelling, identity and power, 255-288. Ed. Alexandra Jaffe, Jannis Androutsopoulos, Mark Sebba \& Sally Johnson. Boston, Berlin: De Gruyter Mouton.

Tabouret-Keller, Andrée (1988). La situation linguistique en Alsace : Les principaux traits de son évolution vers la fin du $\mathrm{XX}^{\mathrm{e}}$ siècle. L'allemand en Alsace, 77-109. Actes du Colloque de Strasbourg. Eds. Adrien Finck \& Marthe Philipp. Strasbourg, Presses Universitaires de Strasbourg / Association des publications près les Universités de Strasbourg.

Tufi, Stefania (2016). Constructing the Self in Contested Spaces: The Case of SlovenianSpeaking Minorities in the Area of Trieste. Negotiating and Contesting Identities in Linguistic Landscapes, 101-116. Eds. Robert Blackwood, Elizabeth Lanza and Hirut Woldemariam. London: Bloomsbury. 
Urbain, Jean-Didier (1988). Récit de mort et mort du récit dans les cimetières. La mort dans le texte, 221-242. Ed. Gilles Ernst. Lyon: Presses Universitaires de Lyon.

Urbatsch, Robert (2014). Nominal Partisanship: Names as Political Identity Signals. PS: Political Science \& Politics 2014.47 (2), 463-467.

Vajta, Katharina (2004) « Nous n'avons plus de langue pour nos fêtes de familles » Le changement de langue dans une famille alsacienne. Göteborg: Acta Universitatis Gothoburgensis.

Vajta, Katharina (2018). Gravestones speak - but in which language? Epitaphs as mirrors of language shifts and identities in Alsace. Journal of Multilingual and Multicultural Development 39(2), 137-154,

Vidor, Gian Marco (2014). Satisfying the mind and inflaming the heart: emotions and funerary epigraphy in nineteenth-century Italy. Mortality 19 (4), 342-360.

Vogler, Bernard, 1993. Histoire culturelle de l'Alsace. Strasbourg, La Nuée Bleue.

Woodthorpe, Kate (2011). Sustaining the contemporary cemetery: Implementing policy alongside conflicting perspectives and purpose. Mortality 16 (3), 259-276.

Wright, Elizabethada A. (2005). Rhetorical Spaces in Memorial Places: The Cemetery as a Rhetorical Memory Place/Space. Rhetoric Society Quarterly 35 (4), 51-81. 